\title{
Cloud distribution evaluated by the WRF model during the EUSO-SPB1 flight
}

\author{
$K$ Shinozaki ${ }^{1,2, *}, S$ Monte $^{1}, S$ Ferrarese $^{1,2}, M$ Manfrin $^{1,2}, M E$ Bertaina $^{1,2}, A$ Anzalone $^{3}, F$ Bisconti $^{2}, A$ Bruno $^{3}, A$ Diaz $^{4}$, \\ $J$ Eser $^{5}, F$ Fenu $^{1,2}, A$ Michel $^{6}, M$ Vrabel $^{7}$, and $L$ Wiencke ${ }^{5}$ on behalf of the JEM-EUSO Collaboration \\ ${ }^{1}$ Università Degli Studi di Torino, Turin, Italy \\ ${ }^{2}$ Istituto Nazionale di Fisica Nucleare - Sezione di Torino, Italy \\ ${ }^{3}$ INAF - Istituto di Astrofisica Spaziale e Fisica Cosmica di Palermo, Italy \\ ${ }^{4}$ IRAP, Université de Toulouse, CNRS, Toulouse, France \\ ${ }^{5}$ Colorado School of Mines, Golden, USA \\ ${ }^{6}$ Observatorie de la Côta d'Azur, Nice, France \\ ${ }^{7}$ Technical University Kosice (TUKE), Kosice, Slovakia
}

\begin{abstract}
EUSO-SPB1 was a balloon-borne mission of the JEM-EUSO (Joint Experiment Missions for Extreme Universe Space Observatory) Program aiming at the ultra-high energy cosmic ray (UHECR) observations from space. We operated the EUSO-SPB1 telescope consisting of $1 \mathrm{~m}^{2}$ Fresnel refractive optics and multianode photomultiplier tubes. With a total of 2304 channels, each performed the photon counting every $2.5 \mu \mathrm{s}$, allowing for spatiotemporal imaging of the air shower events in an $\sim 11^{\circ} \times 11^{\circ}$ field of view. EUSO-SPB1 was the first balloon-borne fluorescence detector with a potential to detect air shower events initiated by the EeV energy cosmic rays. On 24 April 2017 UTC, EUSO-SPB1 was launched on the NASA's Super Pressure Balloon that flew at $\sim 16-33 \mathrm{~km}$ flight height for $\sim 12$ days. Before the flight was terminated, $\sim 27$ hours of data acquired in the air shower detection mode were transmitted to the ground. In the present work, we aim at evaluating the role of the clouds during the operation of EUSO-SPB1. We employ the WRF (Weather Research and Forecasting) model to numerically simulate the cloud distribution below EUSO-SPB1. We discuss the key results of the WRF model and the impact of the clouds on the air shower measurement and the efficiency of the cosmic ray observation. The present work is a part of the collaborative effort to estimate the exposure for air shower detections.
\end{abstract}

\section{Introduction}

Efficient observations of ultra-high energy cosmic rays (UHECRs) are a fundamental requirement for studying their origin. Recently, the leading ground-based experiments, i.e., the Pierre Auger Observatory [1] and the Telescope Array (TA) [2] reported anisotropy of the UHECR arrival direction distribution above $8 \times 10^{18} \mathrm{eV}$ and $\sim 6 \times$ $10^{19} \mathrm{eV}$, respectively. Above the latter energy, the cosmic ray fluxes are as small as 1 per $\mathrm{km}^{2}$ in a century with a steepening spectral index [3]. Typical interpretations of this spectral features are Greisen-Zatsepin-Kuz'min mechanism during the propagation of UHECRs [4, 5] or/and acceleration limit of UHECRs at their sources [3]. To investigate the nature of UHECRs, in particular studies on anisotropy, larger exposures of observation are wanted.

The JEM-EUSO (Joint Experiment Missions for Extreme Universe Space Observatory) program aims at space-based UHECR observations to efficiently increase the exposure [6]. Using an ultra-wide field-of-view (FOV) telescope from a satellite in the low Earth orbit, the fluorescence technique may be applied over a $\sim 10^{5} \mathrm{~km}^{2}$ scale observation area on the Earth's atmosphere that acts as a vast particle calorimeter.

*e-mail: kenji.shinozaki@to.infn.it
In the JEM-EUSO framework, two full-scale missions, i.e., K-EUSO (KLYPVE-EUSO) [7] and POEMMA (Probe Of Extreme Multi-Messenger Astrophysics) [8], are promoted. The former will be based on the International Space Station (ISS). The latter is designed for the stereoscopic observations using two satellites. To test the key technologies developed for JEM-EUSO, we have operated several pathfinders, EUSO-TA [9] on the ground, and the EUSO-Balloon [10] and EUSO-SPB1 [11] as stratospheric balloon payloads. These pathfinders consisted of the $1 \mathrm{~m}^{2}$ Fresnel refractive optics with a photo-detector module (PDM) [12] at the focus. In the near future, the Mini-EUSO pathfinder with a smaller optics will be operated in the ISS [13].

In space-based UHECRs observations, several factors are needed to be taken into account [14]. By pointing the telescope to the Earth, air shower signals are observed on the airglow background light. The variation of such light determines the lowest observable energy of UHECRs. Occasionally, intense artificial light sources such as cities may be seen in the FOV of the telescope. The instantaneous apertures of the UHECR observation decrease in terms of the area, while the trigger algorithm can be designed to be operational for the rest of the FOV. 
Another obstacle is the presence of clouds. Even in such a situation, the detection of air showers is still feasible. Particularly if the height of the clouds is low, the effective area within the FOV of the telescope is not necessarily reduced. A substantial part of the longitudinal shower development including the shower maximum is visible for most of air showers from UHECRs $[14,15]$. As the cloudtop height $(\mathrm{CTH})$ increases, air showers from large zenith angles are still observable. In this way, CTH is a factor to determine the zenith angle range of detectable air showers. In general, monitoring the cloud coverage and the CTH distribution is an important requirement to determine quantities of the area and solid angle acceptance in the instantaneous aperture.

In April 2017, we conducted the EUSO-SPB1 mission to operate the first fluorescence telescope flown to sub-orbital space with the capability of air shower detection. We employed the WRF model [16], a weather forecast model, to simulate the $\mathrm{CTH}$ distribution over the area where the acquired data in the air shower detection mode were retrieved. The presented work is one step towards the goal to estimate the exposure for EUSO-SPB1 and also has applications in space-based UHECR observation.

\section{EUSO-SPB1 flight}

EUSO-SPB1 was the second balloon born prototype following the one night flight of EUSO-Balloon in 2014 over Timmins, Canada. The EUSO-SPB1 telescope consisted of a $1 \mathrm{~m}^{2}$ aperture two-Fresnel-lens optics and a PDM composed of 36 multi-anode photomultiplier tubes (MAPMTs). In total 2304 pixels with $\sim 0.2^{\circ}$ spatial span covered an $\sim 11^{\circ} \times 11^{\circ}$ FOV including small nonefficient part due to the MAPMT spacing. The PDM is sub-divided into nine sets of elementary cells (ECs) each of which composed of $2 \times 2=4$ MAPMTs.

Distinct from other JEM-EUSO pathfinders, EUSOSPB1 was equipped with an autonomous trigger to detect air showers. Moreover, an expected long duration flight up to $\sim 100$ days would allow detection of a few air shower events in the EeV regime.

Prior to the flight of EUSO-SPB1, a field test was carried out at the TA experiment site, Utah, USA to verify and to quantify the trigger and other key functions using a movable laser device and the TA's Central Laser Facility. The clear signal for such laser events, i.e., the detection of the scattered light of laser shots, was detected and the performance of the telescope was evaluated.

On 24 April 2017 UTC, EUSO-SPB1 onboard NASA's Super Pressure Balloon (SPB) was released from the Wanaka airport, New Zealand at latitude (Lat.) $44.72^{\circ} \mathrm{S}$ and longitude (Long.) $169.24^{\circ} \mathrm{E}$. The flight lasted $\sim 292$ hours ( $\sim 12$ days) in South Pacific until EUSOSPB1 was abandoned off the Easter Islands (Lat. $29.38^{\circ} \mathrm{S}$, Long. $106.50^{\circ} \mathrm{W}$ ) on 7 May. This was due to the unexpected gas leakage on the SPB envelope, while the EUSOSPB1 instrument remained fully operational until the end. If it had not happened, EUSO-SPB1 had a capability of floating over $\sim 33 \mathrm{~km}$ above sea level for as long as $\sim 32-$ 47-days as was achieved past SPB flights.

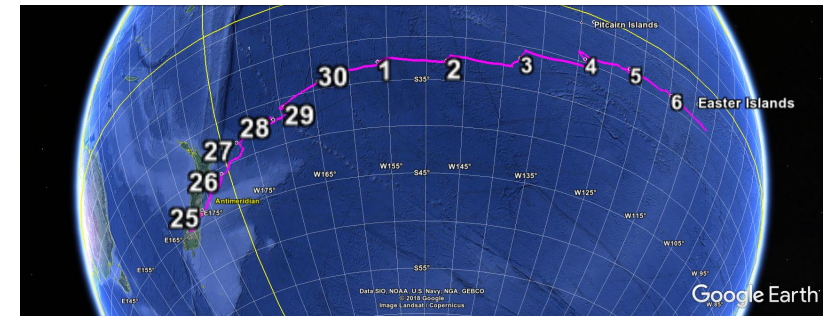

Figure 1. Trajectory of EUSO-SPB1 flight shown on the solid curve. The positions at 12:00 UTC are labeled by the day of the month, April or May.

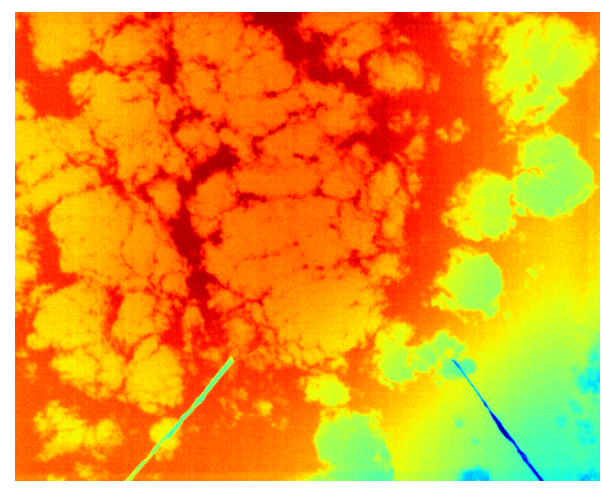

Figure 2. An example of the IR camera image. The raw ADC output is shown without calibration.

Figure 1 shows the trajectory of EUSO-SPB1 by the solid curve on the Google Earth image. Daily positions as of 12:00 UTC are indicated with a label of the day of the month. EUSO-SPB1 resided around the Anti-meridian. This time corresponding to the local solar time was $\sim 0 \mathrm{~h}$, midnight. The operations of the telescope were carried out around these positions on 12 nights.

About 27 hours of the acquired data in the air shower detection mode could be transmitted to the ground during the flight. During those hours, EUSO-SPB1 oversaw various weather situations. To monitor the presence of clouds in the FOV, an infrared (IR) camera [17] was operated in limited times along with the EUSO-SBP1 telescope.

Figure 2 shows an example of the IR camera image taken above a cloudy area. The FOV is $\sim 32^{\circ} \times 24^{\circ}$. The raw data without calibration are displayed. Two stripes suspended from SPB are seen. Further analysis is underway.

As the clouds have high reflectivity to the diffuse light, the background level on the telescope increases in comparison with cloud-free conditions [18]. The cloud pattern and its relative motion with EUSO-SPB1 was also recognised by the telescope itself.

Figure 3 shows images of the cloud passage over the FOV measured by the EUSO-SPB1 telescope. These images are averaged over the 10 adjacent triggers, a time span of $3.2 \mathrm{~ms}$. Each panel corresponds to $\sim 1 \mathrm{~min}$ time interval. 

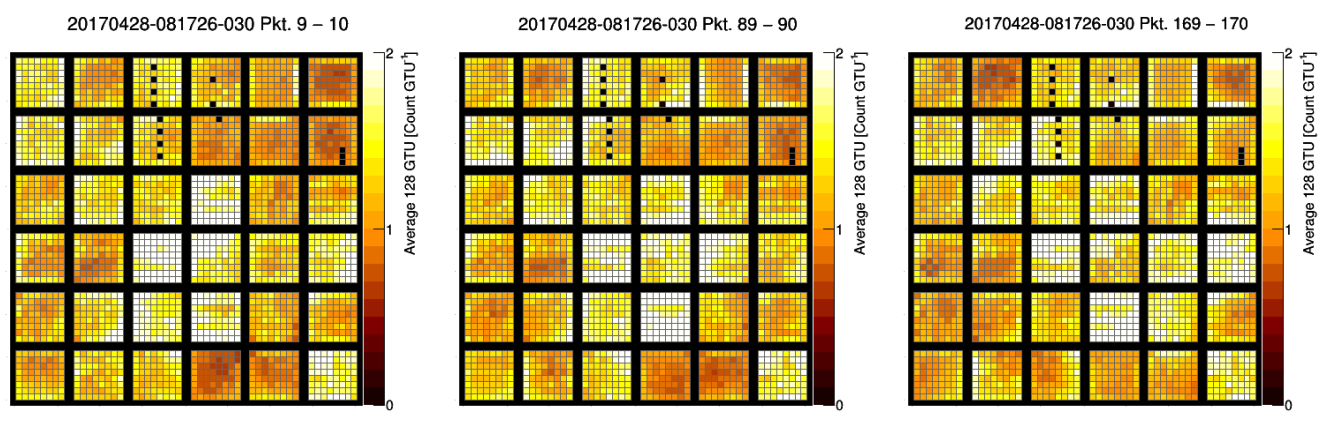

Figure 3. Example of the images of cloud passage over the FOV measured by the EUSO-SPB1 telescope. These images are the averaged 10 adjacent triggers due to the background light fluctuation. Each panel corresponds to $\sim 1$ min time interval.

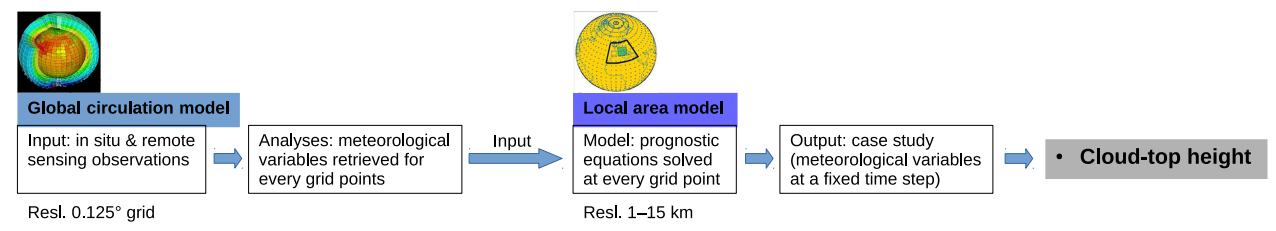

Figure 4. Schematic flowchart of the CTH retrieval with WRF.

\section{Weather Research and Forecast model}

In this section, we briefly describe key functions of the WRF model. Further details can be found in Ref. [19].

Weather Research and Forecasting (WRF) [16] is a mesoscale, non-hydrostatic, fully compressible meteorological model developed by the National Center for Atmospheric Research (NCAR). It is maintained by the collaboration of many institutions such as NOAA. WRF is mainly composed of two parts: WRF Pre-processing System (WPS) and Advanced Research WRF (ARW) solver. For real case simulations as in our case, a set of programs prepare the inputs for real program by interpolating staticterrestrial and dynamic-meteorological data and elaborating initial and boundary conditions. We use a global circulation model (GCM), Integrated Forecasting System (IFS) running at ECMWF (European Centre for Medium-range Weather Forecasts) [20] for the input. Outputs are analysed with post-processor utilities, NCAR Command Language (NCL) [21].

Figure 4 shows a schematic flowchart of the CTH retrieval process for the present work. The GCM output based on both in situ and remote sensing meteorological observations is used as an input of the large area model (LAM). We analysed the output parameters to determine the cloud-top pressure and CTH.

Numerical weather prediction models in general simulate the atmospheric quantities relevant to the CTH without external knowledge such as IR images from weather satellites. The CTH retrieval is based on two main quantities. One is the cloud fraction expressed as the fraction of cloudiness in a model grid-box ranging between 0 and 1. The other is the cloud optical depth, which is estimated from the mixing ratios of water and ice.
In the present work, we ran the WRF model to simulate the cloud fraction in each grid-box within the domain area chosen $\sim 300 \mathrm{~km} \times 300 \mathrm{~km}$ to fit a distance travelled by EUSO-SPB 1 in 24 hours. Outputs are given in 10-minute intervals with $\mathrm{a} \sim 3 \mathrm{~km}$ spatial resolution. We defined CTH at each grid point by the highest level of grid-box with a cloud fraction larger than 0.2 .

Even though EUSO-SPB1 was operated in air shower detection mode for all the nights, the last transmitted data was on 2 May. We thus simulated the cloud fraction distributions on the first 8 nights from 25 April, we set the domain centre to the EUSO-SPB1 position at 12:00 UTC with 50 pressure levels vertically. We ran WRF for any hour in those nights with the retrieved air shower detection mode data. We also simulated for 24 April during ascending of EUSO-SPB1 in daytime to compare with the IR camera data.

\section{Result}

Figure 5 shows examples of the WRF results for 12:00 UTC of 27 April and 29 April on the top and bottom panels, respectively. The left panels show the CTH distributions in a $\sim 300 \mathrm{~km} \times 300 \mathrm{~km}$ domain centred around the EUSO-SPB1 position. The middle show the CTH distribution within a domain. The right visualise the boundaries of the grid-box with a non-zero cloud fraction.

To qualitatively verify the output from the WRF model, we interpreted the images from geostationary weather satellites based on a meteorological analysis. Images from Himawari (Long. $141^{\circ} \mathrm{E}$ ) [22] and GOES (Long. $135^{\circ} \mathrm{W}$ ) [23] were used via Dundee Satellite Receiving Station website [24].

Figure 6 shows examples of the satellite images. The top, middle, and bottom panels show the images at 

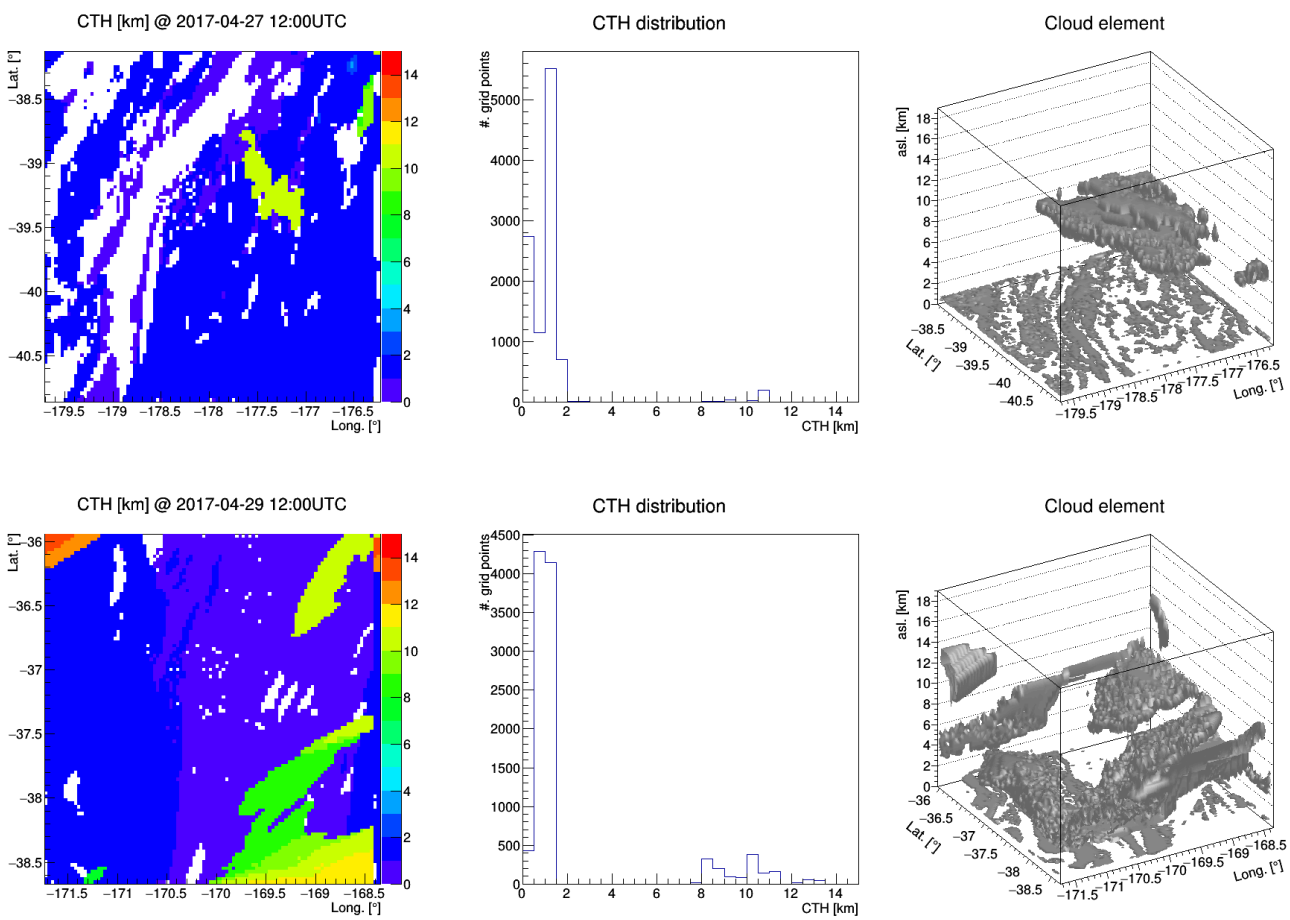

Figure 5. Examples of the WRF results for 12:00 UTC of 27 April and 29 April on the top and bottom panels, respectively. The left panels show the CTH map in a $300 \mathrm{~km}$ domain centred around the EUSO-SPB1 position. The middle show the CTH distribution within a domain. The right visualise the boundaries of the grid-box with a non-zero cloud fraction.

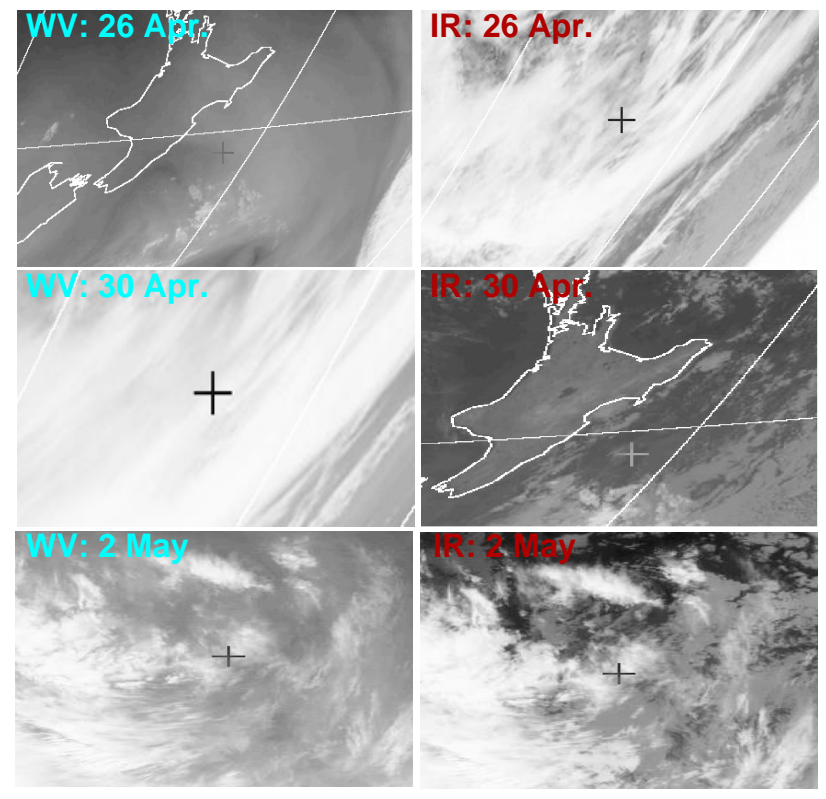

Figure 6. Examples of the satellite images from the top on 26 April, 30 April and 2 May; WV channel on left panels and the IR channel on the right.

12:00 UTC of 26 and 30 April, and 2 May, respectively. The left and right panels correspond to the WV (water vapour) and IR channels, respectively. The 26 April and 30 April images use the Himawari imagery, while the
2 May ones use GOES. Crosses show the EUSO-SPB1 positions.

In the following, a meteorological interpretation on each set of satellite images is summarised. More images are shown on our presentation [25].

- 26 April: the IR channel indicates low-level clouds or fog just west of the EUSO-SPB1 position. The WV channel shows humidity in the upper levels probably without condensation.

- 30 April: Very moist atmosphere in the mid-upper layer and diffused high-level clouds with variable thickness are expected. Additional information from GOES implies possible scattered convective clouds.

- 2 May: Very moist atmosphere is expected. There is widespread cloudiness in mid-level. Scattered highlevel clouds, in some cases thin, are seen. There is a possible convective activity on a 'small' scale along the southwest-northeast direction. By analysing the neighbouring areas, a fairly widespread presence of low clouds is inferred.

Figure 7 summarises the EUSO-SPB1 operational parameters as a function of date together with the WRF retrieved CTH variation on the bottom panel. The flight height of EUSO-SPB1, the average count rates on different ECs shown by different symbols, and cumulative time of data acquisition in air shower detection mode are displayed in the top three panels. The shaded bands indicate daytime or astronomical twilight at EUSO-SPB1. 

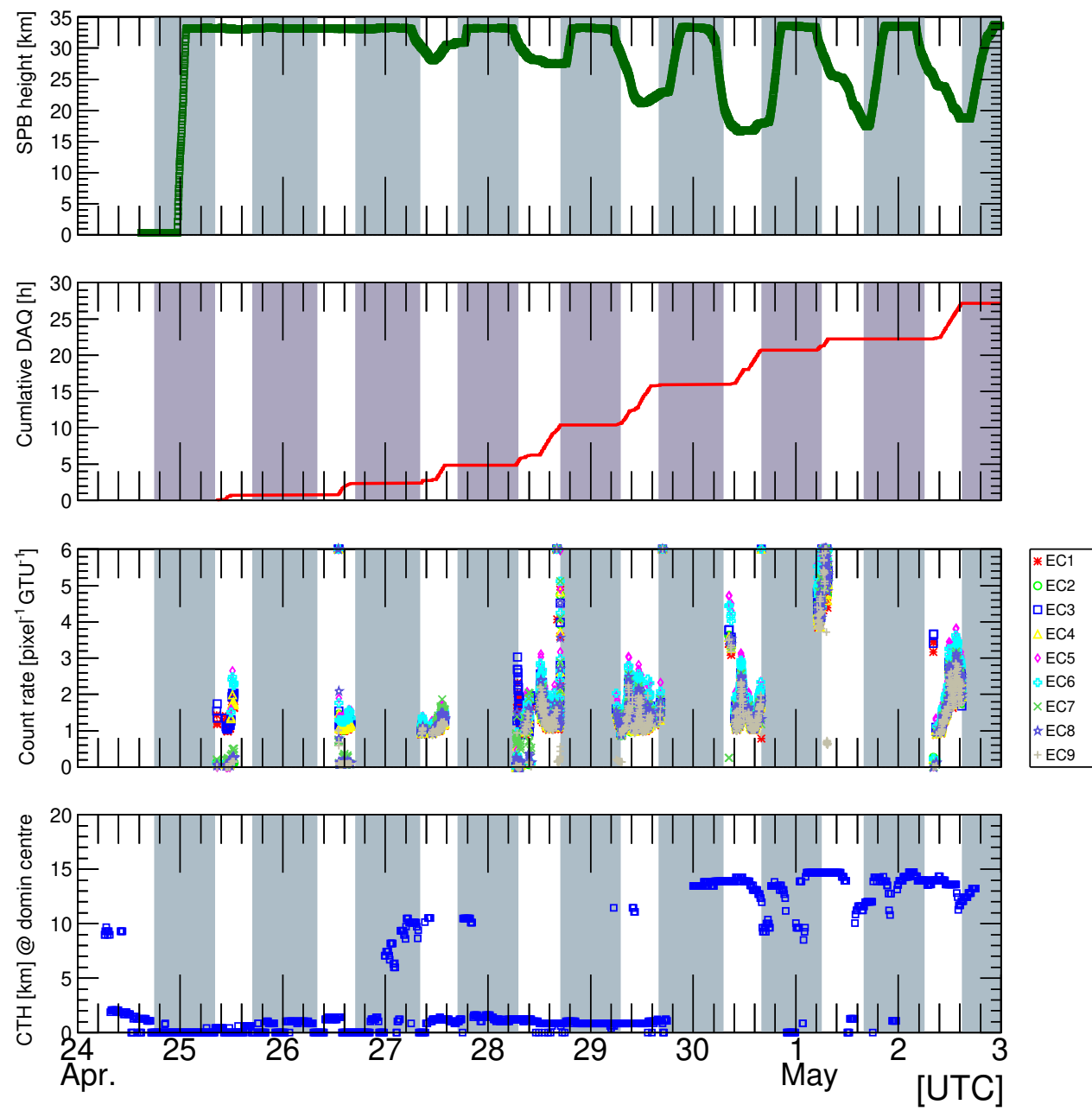

Figure 7. Summary of the EUSO-SPB 1 operational parameters with WRF retrieved CTH on the bottom panel as a function of date. The flight height of EUSO-SPB1, the average count rates on different ECs by different symbols, and cumulative time of data acquisition in air shower detection mode are displayed in the top three panels.

The nominal flight height of EUSO-SPB1 was $\sim 33 \mathrm{~km}$. After 27 April, it tended to drop in the night time. The operation in air shower detection mode was carried out in the flight heights of $\sim 16-33 \mathrm{~km}$.

The average count rates that represent the brightness FOV as background for air shower detections were determined from the data acquired in the air shower detection mode. This information is important to determine the threshold parameters for trigger algorithms. A few hertz trigger rates due to background fluctuations were maintained by the dynamic setting of such parameters [26]. The data are also used in detector response simulations to determine the instantaneous aperture of air shower detection.

The cumulative operation time represents the acquisition time for the data retrieved from EUSO-SPB1. Once the gas leakage was recognised, recovery of the EUSOSPB1 telescope became more unlikely. Thus, we partly modified the data format to allow the downlink of more data. In the end, $\sim 29.9$ hour data, $\sim 2 / 3$ of all acquired data, were retrieved. The data available for the air shower detection mode accounts for $\sim 27.1$ hours.
From the WRF analysis, the cloud fraction in the domain is often large, while the CTH variation was moderately small and $\mathrm{CTH}$ was as low as a few kilometres until 29 April. On 30 April, and 1 and 2 May, CTH was as high as $\sim 15 \mathrm{~km}$. Typical cloud features inferred from the satellite images are in general in agreement with the WRF output. The CTH variation will be used for studying the cloud impact on the air shower detection by simulations.

\section{Summary and discussion}

In the present work, we focus on the application of the WRF model to describe the cloud properties, particularly CTH, during the EUSO-SPB1 flight. This is a part of the comprehensive, collaborative activities to estimate the expected number of air shower events and the exposure for the UHECR observation by EUSO-SPB1.

Simulations for clear atmospheric conditions are primarily relevant to evaluate these values. Currently, the estimation of the exposure during the EUSO-SPB1 flight is underway by the Collaboration using EUSO OffLine [27] 
and the ESAF (EUSO Simulation and Analysis Framework) software [28]. The instantaneous aperture and the effective observation time are the main factors in determining the exposure. The variations of the flight height and the count rate variation both play a role. The former scales the fiducial FOV of air shower detection. The latter determines the thresholds on the trigger algorithm that traces the detectable energy range of UHECRs. These input values to simulations have been investigated and parametrised. The result will be presented in our article in preparation.

The search for air shower events has been also performed by analysing the EUSO-SPB1 data. Such events are characterised by excesses from the background count rates at the confined area within the FOV. They are supposed to move over several microseconds along a 'straight track'. So far, many events with a straight line feature were found that are considered to be low energy primary cosmic ray directly hitting the PDM (see image on Ref. [11]). Detailed analyses on event search will be shortly concluded.

As clouds may attenuate the intensity of fluorescence photons reaching the telescope, this effect needs to be taken into account in simulations and analysis of the EUSO-SPB1 data. The approach by the WRF weather forecast model has been tested in addition to the IR camera data. The present work will be applied to develop a technique to estimate the exposures for future real space-based UHECR observation missions. The further application of the WRF results is ongoing. For instance, the CTH variation is used to model the test cloud distribution for ESAF.

For 2022, another SPB flight for the UHECR observation, i.e., the EUSO-SPB2 mission has been foreseen. This will be another balloon-borne pathfinder for K-EUSO and POEMMA. For the science case of UHECR physics that will be investigated by these full-scale space-based missions, one needs to primarily determine the energy spectrum and arrival direction distribution. Both require the precise exposure determination through the varying conditions such as the background light level, cloud distribution in the FOV, the location along orbit etc. EUSO-SPB1 provided the first platform to study such continuously varying situations together with its air shower detection capability. Experience gathered and method developed such as the use of the weather forecast model will be applied in future missions.

\section{Acknowledgments}

This work was partially supported by NASA grants NNX13AH54G, NNX13AH52G, the French Space Agency (CNES), the Italian Space Agency through the ASI INFN agreement n. 2017-8-H.0, the Italian Ministry of Foreign Affairs and International Cooperation, the Basic Science Interdisciplinary Research Projects of RIKEN and JSPS KAKENHI Grant (22340063, 23340081, and 24244042), and the Deutsches Zentrum für Luft und Raumfahrt, and the Helmholtz Alliance for Astroparticle Physics HAP' funded by the Initiative and Networking Fund of the Helmholtz Association (Germany). We acknowledge the NASA Balloon Program Office and the Columbia Scientific Balloon Facility and staff for extensive support, the Telescope Array Collaboration for the use of their facilities in Utah.

\section{References}

[1] The Pierre Auger Coll., Science 357 (2017) 1266.

[2] RU Abbasi et al., Astrophys. J. 862, 92 (2018).

[3] BR Dawson, M Fukushima, and P Sokolsky, Prog. Theor. Exp. Phys., 12 (2017) 12A107.

[4] K Greisen, Phys. Rev. Lett. 16 (1966) 748.

[5] GT Zatsepin and VA Kuz'min, J. Exp. Theor. Phys. Lett. 4 (1966) 78.

[6] ME Bertaina et al., in these proceedings.

[7] P Klimov et al., Proc. of 35th Int. Cosmic Ray Conf. (Busan, 2017) Pos (ICRC2017) 412.

[8] A Olinto et al., Proc. of 35th Int. Cosmic Ray Conf. (Busan, 2017) Pos (ICRC2017) 542.

[9] F Bisconti et al., in these proceedings.

[10] G Abedellaoui et al., J. Inst. 13 (2018) P05023.

[11] L Wiencke et al., Proc. 35th Int. Cosmic Ray Conf., Pos (ICRC2017) 1079 (2017).

[12] The JEM-EUSO Coll., Exp. Astron. 40 (2015) 19.

[13] F Capel et al., Adv. Space Res. 62 (2018) 2954.

[14] JH Adams et al., Astropart. Phys., 44, 76 (2013).

[15] The JEM-EUSO Coll., Exp. Astron., 40, 135 (2015).

[16] National Center for Atmospheric Research (NCAR), 'WRF the Weather Research and Forecasting Model', http://www.mmm.ucar.edu/weatherresearch-and-forecasting-model

[17] L Allen et al., Proc. 35th Int. Cosmic Ray Conf. (Busan, 2017) Pos (ICRC2017) 436.

[18] S Mackovjak et al., Proc. 34th Int. Cosmic Ray Conf. (The Hague, 2015), Pos (ICRC2015) 443.

[19] S Monte, 'Sensitivity of WRF meteorological model in retrieving Cloud Top Height and application to EUSO-SPB1 experiment', Master Thesis, University of Turin (2018).

[20] ECMWF | Advancing global NWP through international collaboration, https://www.ecmwf.int/

[21] CISL's NCAR Command Language (NCL), http://www.ncl.ucar.edu/

[22] Japan Meteorological Agency (JMA), 'Himawari 8/9 Operational Information' http://www.data.jma.go.jp/mscweb/en/operation8

[23] NOAA "Geostationary Satellite Server", http://www.goes.noaa.gov

[24] Dundee Satellite Receiving Station, http://www.sat.dundee.ac.uk/

[25] K Shinozaki et al., the poster presentation in UHECR2018 conference.

[26] J Bayer et al., Proc. 35th Int. Cosmic Ray Conf. (Busan, 2017), Pos (ICRC2017) 443.

[27] T Paul et al., Proc. 35th Int. Cosmic Ray Conf., Pos (ICRC2017) 443.

[28] C Berat et al. Astropart. Phys., 33, 221 (2010). 